\title{
High-resolution grating interferometer for phase-contrast imaging at PETRA III
}

\begin{abstract}
A. Hipp, J. Moosmann, J. Herzen, J. U. Hammel, A. Schreyer, et al.
\end{abstract}

A. Hipp, J. Moosmann, J. Herzen, J. U. Hammel, A. Schreyer, F. Beckmann, "High-resolution grating interferometer for phase-contrast imaging at PETRA III," Proc. SPIE 10391, Developments in X-Ray Tomography XI, 1039108 (25 September 2017); doi: 10.1117/12.2273892

SPIE Event: SPIE Optical Engineering + Applications, 2017, San Diego, California, United States 


\title{
High-resolution grating interferometer for phase-contrast imaging at PETRA III
}

\author{
A. Hipp ${ }^{a}$, J. Moosmann ${ }^{a}$, J. Herzen ${ }^{b}$, J. U. Hammel ${ }^{a}$, A. Schreyer ${ }^{c}$ and F. Beckmann ${ }^{a}$ \\ ${ }^{a}$ Helmholtz-Zentrum Geesthacht, Max-Planck-Straße 1, Geesthacht, Germany; \\ ${ }^{b}$ Technische Universität München, James-Frank Straße 1, Garching, Germany; \\ ${ }^{c}$ European Spallation Source (ESS), Tunavågen 24, Lund, Sweden
}

\begin{abstract}
Phase-contrast imaging has proven to be a valuable tool when investigating weak absorbing materials like soft tissue, due to its increased contrast compared to conventional absorption-contrast imaging. While propagationbased phase-contrast is an ideal tool to achieve highest resolution at a good contrast for almost not-absorbing material, it quickly comes to its limitations on applications demanding for a high dynamic range in contrast. For those applications grating-based phase-contrast is the tool of choice, although it lacks of spatial resolution compared to inline phase-contrast or attenuation-based microCT. To reduce this gap in spatial resolution we equipped the two PETRA III beamlines P05 and P07 with a customized set of mechanics to maximize the performance of the interferometer. After latest optimization steps our system allows for phase-contrast measurements in a continuous energy range between $10 \mathrm{keV}$ and $80 \mathrm{keV}$. Dependent on investigated material and energy the setup is capable to achieve a spatial resolution of $5 \mu \mathrm{m}$ on a field of view of $6.5 \mathrm{~mm}$. We will present our implementation of grating-based phase-contrast computed tomography for fast and high-resolution measurements at the PETRA III along with its recent optimization, and demonstrate its performance based on different kinds of applications.
\end{abstract}

Keywords: Phase Contrast, Grating Interferometer, X-ray Imaging, Synchrotron Radiation, Microtomography

\section{INTRODUCTION}

Differential phase-contrast imaging (DPC) has proven to be a valuable tool when investigating weak absorbing materials like soft tissue, due to its increased contrast compared to conventional absorption-contrast imaging. ${ }^{1-3}$ The most common type of grating interferometer consists of two gratings, a phase grating and an analyzer grating. ${ }^{3,4}$ The performance of such an interferomater is mainly limited by the feasible aspect ratio of the analyzer-grating structures. The limitation of structure height also limits the possible energy range and the period of the grating defines the smallest possible pixel size of the detector. To achieve grating structures with a high aspect ratio is still the critical part in grating production. ${ }^{5,6}$ In contrast to this, a single-grating interferometer comes with several advantages: Due to the absence of an analyzer grating, the photon flux at the detector plane is increased by almost a factor of two, resulting in a shorter acquisition time, and at the same time allows for using this type of interferometer at any photon energy supported by the phase grating and propagation distance. Additionally, the setup itself is more stable and easily adjustable. Most important equipment for this type of interferometer is a detector system with a high spatial resolution, that allows to resolve the interference pattern directly. The microtomography endstation at the beamline P05, ${ }^{7,8}$ operated by the Helmholtz-Zentrum Geesthacht at the synchrotron radiation storage ring PETRA III at DESY (Hamburg, Germany), reaches an effective pixel size between $0.3 \mu \mathrm{m}$ and $2.4 \mu \mathrm{m}$. This is an ideal basis for high-resolution phase-contrast imaging using a single-grating interferometer. Here we will present our investigations of a singlegrating interferometer and its performance comparison to a conventional double-grating setup on the basis of a test phantom tomography.

Further author information:

A. Hipp: E-mail: alexander.hipp@hzg.de, Telephone: +49 (0)40 89985301

Developments in X-Ray Tomography XI, edited by Bert Müller, Ge Wang, Proc. of SPIE Vol. 10391, $1039108 \cdot$ (c) 2017 SPIE · CCC code: $0277-786 X / 17 / \$ 18 \cdot$ doi: $10.1117 / 12.2273892$ 


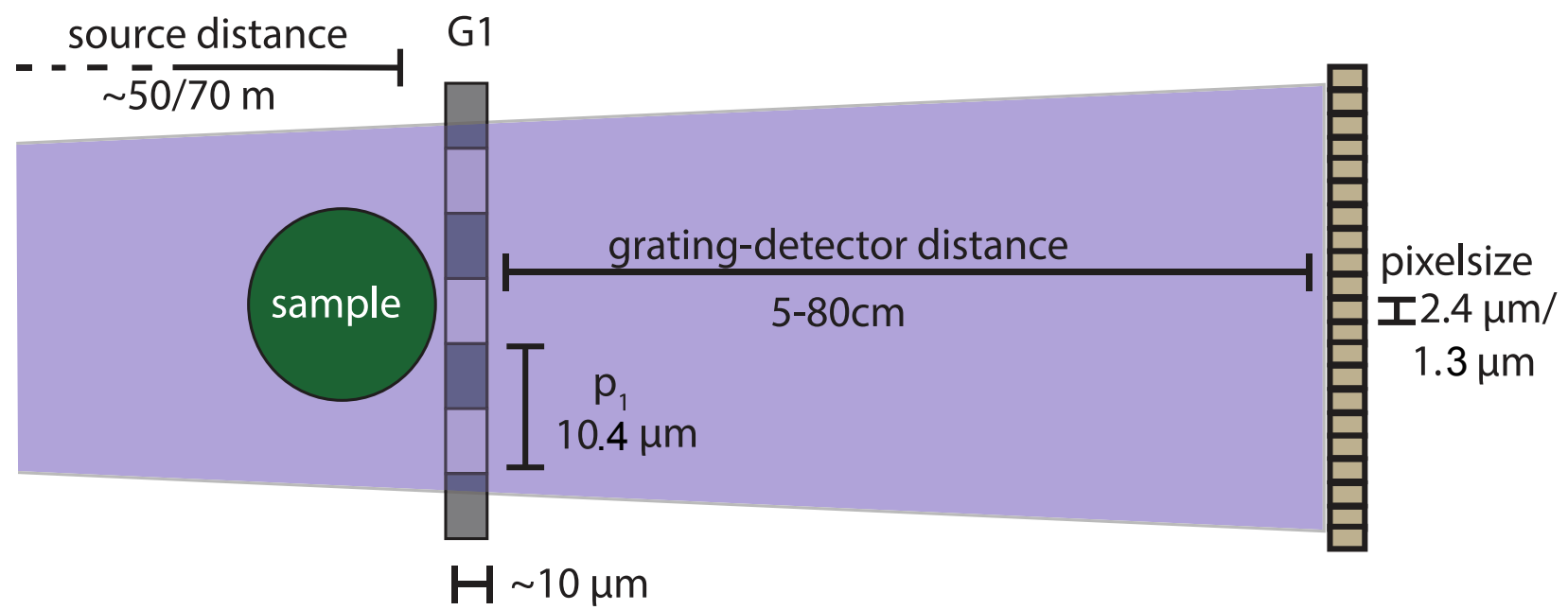

(Ni)

Figure 1: Sketch of the single-grating setup instrumentation. The phase grating (nickel) with a period of $10 \mu \mathrm{m}$ and a height of $10.4 \mathrm{\mu m}$ is placed behind the sample. The propagation distance can be adjusted between $5 \mathrm{~cm}$ and $80 \mathrm{~cm}$. Dependent on the chosen detector system the effective pixel size can be either $2.4 \mu \mathrm{m}(\mathrm{CCD})$ or $1.3 \mu \mathrm{m}(\mathrm{CMOS})$.

\section{INSTRUMENTATION}

All gratings used for the interferometer setups were fabricated by the Institute for Microstructure Technology (IMT) of the Karlsruhe Institute of Technology (KIT) using the LIGA technology. ${ }^{9}$ As substrate, 4"-Si-wafers with a thickness of $200 \mathrm{\mu m}$ were used for the phase-gratings. The analyzer grating for the double-grating setups used a $525 \mu \mathrm{m}$ thick 4"-wafer as substrate. The grating structures were made of nickel for the phase gratings and gold for the analyzer grating. As detector system two different kinds of camera, one CCD-based and one CMOS-based, are used in combination with a optical magnification. The effective pixel sizes are $2.4 \mu \mathrm{m}(\mathrm{CCD})$ and $1.3 \mu \mathrm{m}$ (CMOS).

\subsection{Single Grating}

The single-grating setup for all measurements consists of a phase-grating with period $p_{1}=10 \mu \mathrm{m}$ and a structure height of $10.4 \mu \mathrm{m}$, inducing a phase-shift of $\pi / 2$ at its design energy of $60 \mathrm{keV}$. Due to the similarity of the interference pattern related to a phase-shift of $\pi / 2$ and $3 \pi / 2$, the grating is also suitable for an experiment at $20 \mathrm{keV}$. For all other energies the effective structure height can be adjusted appropriately by tilting the grating towards beam direction by a certain angle $\alpha$.

$$
\alpha(E)= \begin{cases}\arccos (20 \mathrm{keV} / E) & \text { for } 20 \mathrm{keV} \leq E \leq 60 \mathrm{keV} \\ \arccos (60 \mathrm{keV} / E) & \text { for } E \geq 60 \mathrm{keV}\end{cases}
$$

An important factor for a satisfying performance is an adequate ratio of interference pattern period $p_{1}$ to pixel size $s$, which should satisfy:

$$
\frac{p_{1}}{s} \geq 4
$$




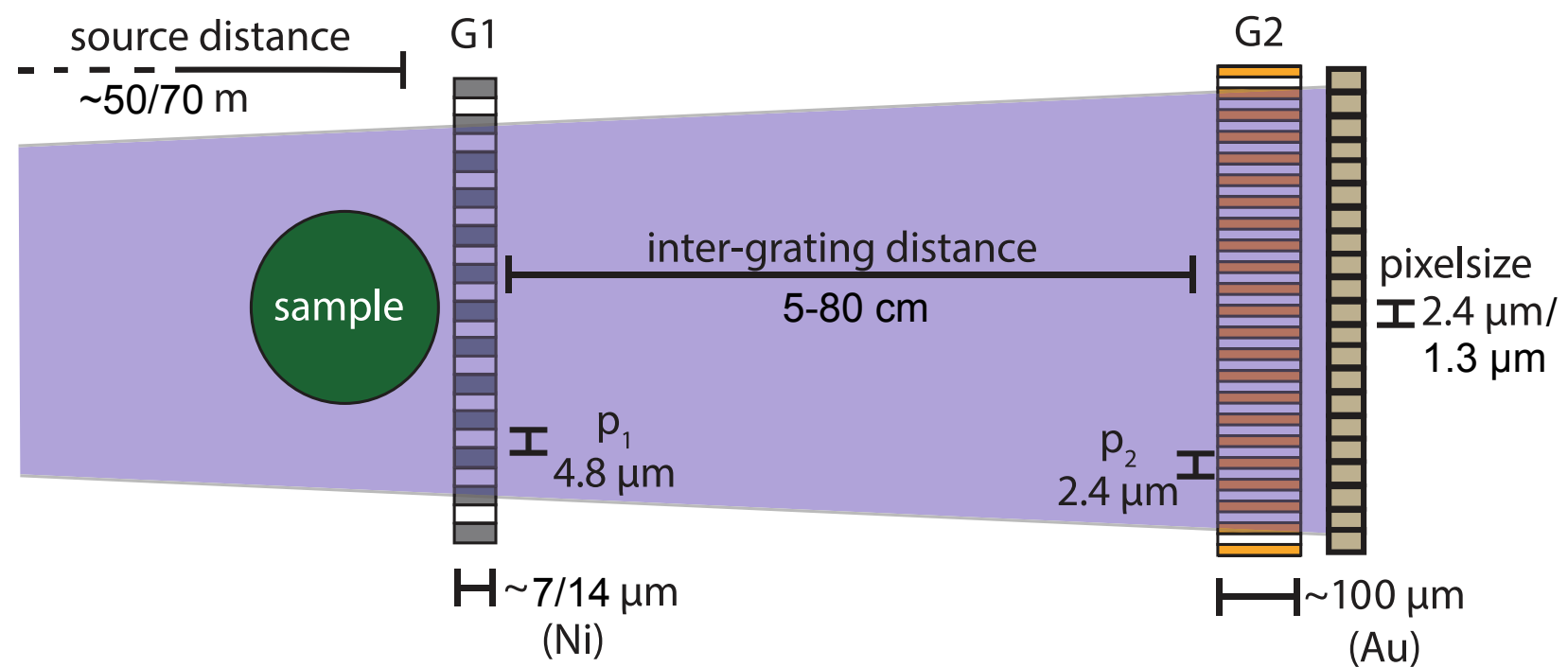

Figure 2: Sketch of the double-grating setup instrumentation. The phase grating (nickel) with a period of $4.8 \mu \mathrm{m}$ is placed behind the sample. Its height is $7 \mu \mathrm{m}$ respectively $14 \mu \mathrm{m}$. The analyser grating (gold) has a period of $2.4 \mu \mathrm{m}$ with a structure height of $\approx 100 \mu \mathrm{m}$. The inter-grating distance can be adjusted between $5 \mathrm{~cm}$ and $80 \mathrm{~cm}$. Dependent on the chosen detector system the effective pixel size can be either $2.4 \mu \mathrm{m}$ or $1.3 \mu \mathrm{m}$.

With the effective pixel sizes of $2.4 \mu \mathrm{m}$ and $1.3 \mu \mathrm{m}$, the grating of $10 \mu \mathrm{m}$ period fits to equation 2 and can be resolved directly with both camera systems. The propagation distance behind the phase-grating is adjusted by placing the detector tower and thereby the scintillator screen to the required position. In total the detector tower can be placed up to $100 \mathrm{~cm}$ from phase-grating. Due to the limited travel distance of the detector tower, the energy range of the single-grating setup limited by the reachable propagation distance. So far, the highest energy successfully used with this setup was $80 \mathrm{keV}$, but with a very short propagation distance of only a quarter of the first fractional Talbot distance. For higher energies or a larger relative propagation distance a phase grating with a smaller period has to be used. Figure 1 presents a sketch of the single-grating setup and its dimensions.

\subsection{Double Grating}

Dependent on the energy range, the double grating interferometer is set up with two different phase gratings. Both gratings have a period of $4.8 \mu \mathrm{m}$, with structure heights of $7 \mu \mathrm{m}$ and respectively $14 \mu \mathrm{m}$. These structure heights correspond to design energies of $20 \mathrm{keV}$ and $40 \mathrm{keV}$. Other energies then these two can be applied by tilting the appropriate grating towards the beam direction by a certain angle $\alpha$ :

$$
\alpha(E)= \begin{cases}\arccos (6.7 \mathrm{keV} / E) & \text { for } 6.7 \mathrm{keV} \leq E \leq 20 \mathrm{keV} \\ \arccos (20 \mathrm{keV} / E) & \text { for } 20 \mathrm{keV} \leq E<40 \mathrm{keV} \\ \arccos (40 \mathrm{keV} / E) & \text { for } E \geq 40 \mathrm{keV}\end{cases}
$$

Here, the energy range between $6.7 \mathrm{keV}$ and $20 \mathrm{keV}$ is accessed by the $7 \mu \mathrm{m}$ high grating, giving a $3 \pi / 2$-shift. The analyser grating for all setups has a period of $2.4 \mu \mathrm{m}$ and structure height of $\approx 100 \mu \mathrm{m}$. To achieve such a high aspect ratio, the grating was produced using the 'sunray'-design. ${ }^{6}$ For a tilted setup the analyser grating was tilted by the same angle to keep the inter-grating distance constant across the full field of view. The tilt of the analyser grating also results in a higher effective structure height, which comes with a better absorption. With the analyser grating directly mounted to the detector tower, the inter-grating distance was adjusted by moving the detector tower. This setup was successfully utilised in the energy range from $20 \mathrm{keV}$ up to $100 \mathrm{keV}$. ${ }^{10}$ Figure 2 gives a sketch and dimensions for the double-grating interferometer setups. 
Table 1: Experimental parameters for the different measurements with the single-grating setup $S G$ and the double-grating setups $D G_{1}, D G_{3}$. Phase grating period $p_{1}$, analyzer grating period $p_{2}$, phase shift $\Phi$, phase grating height $h_{1}$, analyzer grating height $h_{2}$, propagation distance $d$ and visibility $V$.

\begin{tabular}{|c|c|c|c|c|}
\hline & & $S G$ & $D G_{1}$ & $D G_{3}$ \\
\hline & $p_{1}$ & $10 \mu \mathrm{m}$ & $4.8 \mu \mathrm{m}$ & $4.8 \mu \mathrm{m}$ \\
\hline & $p_{2}$ & - & $2.4 \mu \mathrm{m}$ & $2.4 \mu \mathrm{m}$ \\
\hline & $\Phi$ & $3 \pi / 2$ & $\pi$ & $\pi$ \\
\hline \multirow{4}{*}{ 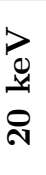 } & $h_{1}$ & $10.4 \mu \mathrm{m}$ & $7 \mu m$ & $7 \mu m$ \\
\hline & $h_{2}$ & - & $\approx 100 \mu \mathrm{m}$ & $\approx 100 \mu \mathrm{m}$ \\
\hline & $d$ & $40 \mathrm{~cm}$ & $4.6 \mathrm{~cm}$ & $13.9 \mathrm{~cm}$ \\
\hline & $V$ & $8 \%$ & $49 \%$ & $38 \%$ \\
\hline \multirow{4}{*}{$\begin{array}{l}P_{0} \\
\stackrel{y}{4} \\
\stackrel{ }{\forall}\end{array}$} & $h_{1}$ & $20.8 \mu m$ & $14 \mu m$ & $14 \mu m$ \\
\hline & $h_{2}$ & - & $\approx 100 \mu \mathrm{m}$ & $\approx 100 \mu \mathrm{m}$ \\
\hline & $d$ & $80 \mathrm{~cm}$ & $9.3 \mathrm{~cm}$ & $27.9 \mathrm{~cm}$ \\
\hline & V & $6 \%$ & $25 \%$ & $16 \%$ \\
\hline
\end{tabular}
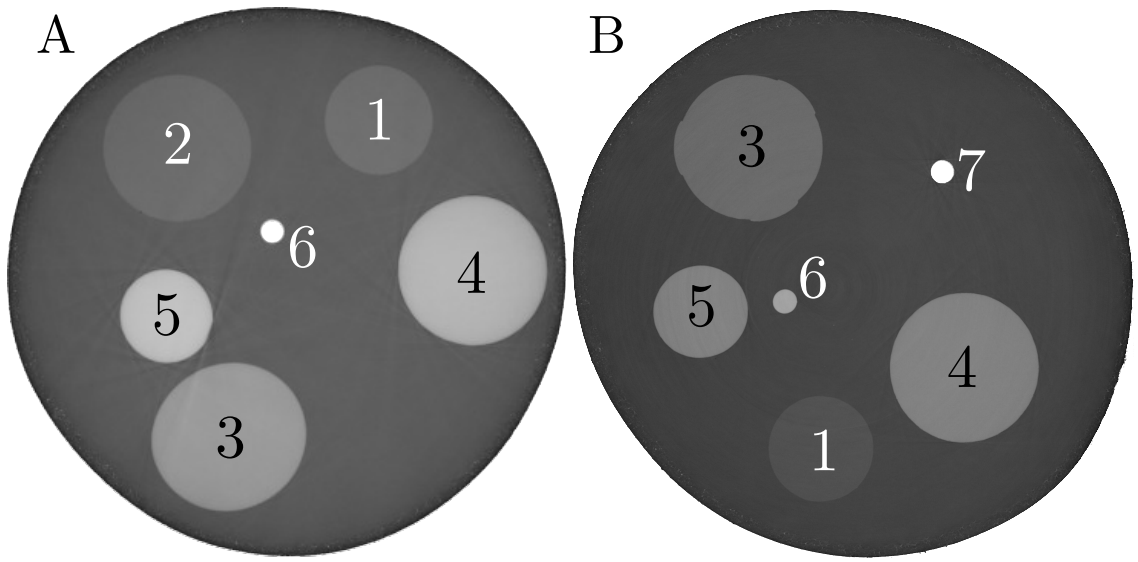

Figure 3: Cross section of the two used phantom samples used at $20 \mathrm{keV}$ (A) and $40 \mathrm{keV}$ (B). 1: PMMA, 2: PA 6.6, 3: Magnesium, 4: PCTFE, 5: PTFE, 6: Aluminium, 7: Titanium.

\section{EXPERIMENTAL METHOD}

The tomography experiments were conducted at a photon energy of $20 \mathrm{keV}$ and $40 \mathrm{keV}$ using both single-grating and a double-grating setup. The setup parameters are listed in Table 1. The propagation distance was set to half of the first fractional Talbot distance for the single-grating setup to avoid too strong image doubling from the beamsplitter characteristic. ${ }^{11}$ The inter-grating distance for the double-grating interferometer was set to the third fractional Talbot distance. At this distance strong artefacts are expected, therefore the measurements were repeated at the first fractional Talbot distance. To achieve a phase-grating height of $20.8 \mu \mathrm{m}$ for the $S G$-setup the grating was tilted by an inclination angle of $60^{\circ}$.

For the measurements at $20 \mathrm{keV}$ and $40 \mathrm{keV}$ two different cameras were used. For the $20 \mathrm{keV}$ measurements a CCD-based camera with an effective pixel size of $2.4 \mu \mathrm{m}$ and a dynamic range of 16 bit was used, whereas the measurements at $40 \mathrm{keV}$ were performed using a CMOS-based camera with an effective pixel size of $1.3 \mu \mathrm{m}$ and a dynamic range of 12 bit. For each tomogram a series of 900 projections were taken with a sample rotation over $180^{\circ}$. For each projection a series of four images (three phase steps) was taken. Although the minimum number of phase steps needed to determine the phase of the stepping curve is only two, measurements with only three images per phase-scan where not reliable. 
Table 2: List of the used materials for the test phantoms, describing their diameter, electron density $\rho_{e}$ and refractive index decrement $\delta$.

\begin{tabular}{lcccc}
\hline Material & Diameter $[\mathrm{mm}]$ & $\rho_{e}\left[10^{23} / \mathrm{cm}^{3}\right]$ & $\delta(20 \mathrm{keV})\left[10^{-7}\right]$ & $\delta(40 \mathrm{keV})\left[10^{-7}\right]$ \\
\hline PA $6.6^{\mathrm{a}}$ & 1.6 & 3.77 & 6.46 & \\
PMMA $^{\mathrm{b}}$ & 1 & 3.87 & 6.67 & 1.67 \\
$\mathrm{Mg}$ & 1.6 & 5.17 & 8.95 & 2.23 \\
PCTFE $^{\mathrm{c}}$ & 1.6 & 6.25 & 10.7 & 2.66 \\
PTFE $^{\mathrm{d}}$ & 1 & 6.24 & 11.0 & 2.74 \\
$\mathrm{Al}$ & 0.25 & 7.83 & 13.6 & 3.38 \\
Ti $_{\text {HMA }}^{\mathrm{e}}$ & 0.25 & 12.45 & & 5.38 \\
\hline
\end{tabular}

\section{Phantom Sample}

Different polymer and metal rods were embedded in a polymer matrix as phantom samples. The designed diameter of the phantoms is $6 \mathrm{~mm}$ in order to match the field of view of the detector system, leaving some free space around the sample for background corrections and noise analysis. Each phantom was fabricated with a compilation of 6 different rods from the materials listed in Table 2. The table list the values for the electron density $\rho_{e}$ and the corresponding refractive index decrement $\delta$ for the investigated energies. The materials and their respective diameters were chosen to cover a wide range of attenuation and phase-shift cross section, and meanwhile keeping the maximum attenuation low enough to minimize the possible starvation effects. ${ }^{12}$

\section{RESULTS}

\subsection{Signal to Noise Ratio}

For each material, the mean value of the retrieved $\delta$-values and the signal to noise ratio (SNR) were calculated. The SNR value is conventionally calculated by taking the average over the signal and the standard deviation of an uniform background. However, the noise within the areas of the materials is strongly dependent on their properties. Especially the scattering caused by the sample has strong effects as it directly negatively influences the visibility which in return deteriorates the image quality. Therefore the noise values were taken from within the material instead of the background. This approach is applicable, because the object composed of homogeneous materials where the noise level is not compromised by inhomogeneity features from within the object.

For all tomographic reconstructions three slices equally separated were analysed. On each slice the mean value of each material and the correspondent noise value was determined in a square field in the middle of the material. Each area was $60 \times 60$ pixels large. This relatively small area had to be chosen with respect to the small diameter of the Aluminium and Titanium rod.

Table 3 list the results from the measurements at $20 \mathrm{keV}$. The retrieved $\delta$-values and SNR are given. For all materials the best results of the mean $\delta$-value (compare Table 2 are obtained by the double-grating interferometer at the first fractional Talbot distance $\left(D G_{1}\right)$. The values from the double-grating setup at the third fractional Talbot distance $\left(D G_{3}\right)$ and from the single-grating setup $(S G)$ have a relative large deviation from the theoretical values. One possible reason for this lies in the phase-wrapping artefacts, which occur at the borders between the different materials. Phase-wrapping in the single projections not only affects the reconstruction by generating streak artefacts tangential to the materials border, but also leading to cupping artefacts, that disturb the

\footnotetext{
${ }^{\text {a }}$ Polyamid, Nylon 6.6

${ }^{\mathrm{b}}$ Polymethylmethacrylat

${ }^{\mathrm{c}}$ Polychlortrifluorethylen

${ }^{\mathrm{d}}$ Polytetrafluorethylen

${ }^{\text {e}}$ Hot melt adhesive, composition not known.

The values listed in the table correspond to Polyethylene, a common base material of HMA.
} 
Table 3: Signal quality comparison for a single-grating (SG) and a double-grating interferometer at the first $\left(D G_{1}\right)$ and at the third $\left(D G_{3}\right)$ fractional Talbot distance. Mean values of material dependent $\delta$-values and related signal to noise ratios for measurements at $20 \mathrm{keV}$ and $40 \mathrm{keV}$ are given.

\begin{tabular}{|c|c|c|c|c|c|}
\hline & & \multicolumn{2}{|c|}{$20 \mathrm{keV}$} & \multicolumn{2}{|c|}{$40 \mathrm{keV}$} \\
\hline & Material & $\delta\left[10^{-} 7\right]$ & SNR & $\delta\left[10^{-} 7\right]$ & SNR \\
\hline \multirow{7}{*}{$S G$} & PMMA & 6.26 & 79.62 & 1.65 & 89.23 \\
\hline & PA 6.6 & 6.26 & 75.10 & - & - \\
\hline & $\mathrm{Mg}$ & 8.50 & 109.13 & 2.22 & 100.42 \\
\hline & PCTFE & 9.75 & 123.11 & 2.67 & 148.45 \\
\hline & PTFE & 10.50 & 134.52 & 2.69 & 140.89 \\
\hline & $\mathrm{Al}$ & 12.56 & 119.65 & 3.34 & 180.10 \\
\hline & $\mathrm{Ti}$ & - & - & 5.32 & 218.43 \\
\hline \multirow{7}{*}{$D G_{1}$} & PMMA & 6.75 & 119.80 & 1.69 & 131.74 \\
\hline & PA 6.6 & 6.65 & 138.59 & - & - \\
\hline & $\mathrm{Mg}$ & 8.85 & 167.82 & 2.25 & 162.44 \\
\hline & PCTFE & 10.19 & 171.75 & 2.66 & 166.39 \\
\hline & PTFE & 10.96 & 184.75 & 2.80 & 180.43 \\
\hline & $\mathrm{Al}$ & 12.81 & 255.40 & 3.43 & 144.10 \\
\hline & $\mathrm{Ti}$ & - & - & 5.32 & 218.43 \\
\hline \multirow{7}{*}{$D G_{3}$} & PMMA & 6.02 & 93.90 & 1.60 & 117.23 \\
\hline & PA 6.6 & 5.80 & 105.79 & - & - \\
\hline & $\mathrm{Mg}$ & 8.01 & 152.13 & 2.08 & 137.76 \\
\hline & PCTFE & 9.45 & 181.58 & 2.53 & 165.76 \\
\hline & PTFE & 9.73 & 217.39 & 2.64 & 153.45 \\
\hline & $\mathrm{Al}$ & 11.64 & 242.74 & 3.17 & 168.73 \\
\hline & $\mathrm{Ti}$ & - & - & 4.8 & 154.60 \\
\hline
\end{tabular}

whole grey-level distribution, which are similar to artefacts occurring at region-of-interest measurements for conventional $\mu \mathrm{CT} .{ }^{13}$

A two dimensional cross section of the reconstructed volumes is presented in figure 4. The artefacts from phase-wrapping can be detected at the sample border and appears like a bell-shaped envelope on the reconstructed slices. This envelope induces a false grey level distribution of the sample, especially obvious in the border region.

To have a systematic comparison of the quantitative results, the experiment was repeated with a slightly different phantom at a photon energy of $40 \mathrm{keV}$. At this energy the corruption from phase-wrapping is negligible for the SG measurement, which can be seen from the obtained $\Delta \delta$-values and the increased SNR, also listed in Table 3. The measurement using the $D G_{1}$ again show very good agreement to the literature values and slightly improved SNR values to those from the same measurement at $20 \mathrm{keV}$. The $\delta$-values retrieved from the $D G_{1}$ measurement are again in very good agreement with the literature values and the SNR values are comparable to those from the $20 \mathrm{keV}$ measurement. For the $D G_{3}$ measurement a strong improvement in the accuracy of the $\delta$ values is recognisable, although the deviation from the literature values is still strong. This indicates that the measurement still suffers from phase-wrapping and can be proven by the two dimensional cross section shown in figure 5 .

\subsection{Spatial Resolution}

For this study the spatial resolution analysis was performed on the three-dimensional datasets. The structure of the phantom with its well separated materials allows to determine the spatial resolution using the well defined interface between the individual rods and the filling material. This interface represents a step function that can be analysed via an MTF. For each rod of the test phantom, a line starting in the filling material and ending in the 


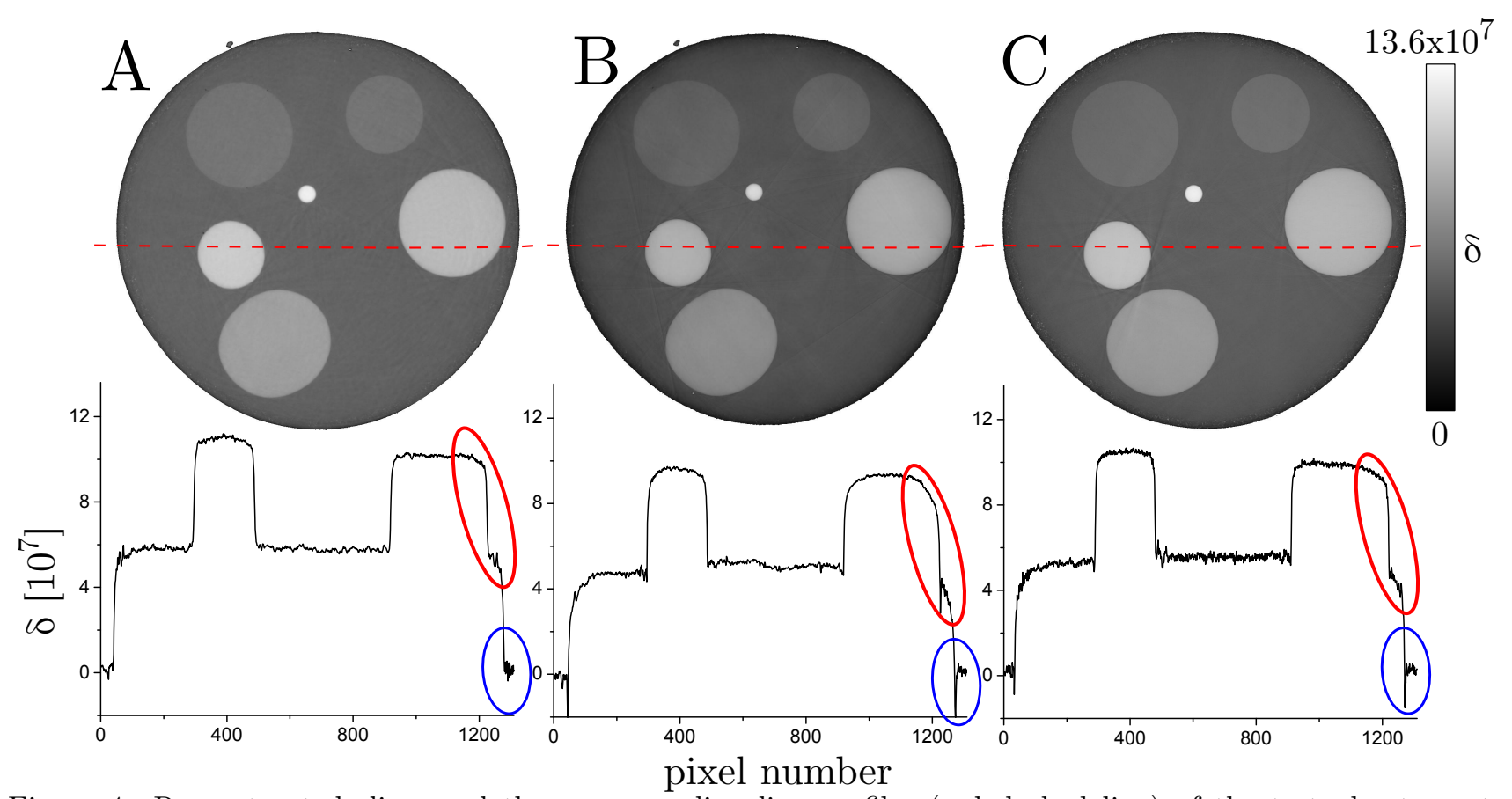

Figure 4: Reconstructed slices and the corresponding line profiles (red dashed line) of the test phantom at the different setups. A: Double-grating setup at the first fraction Talbot distance $\left(D G_{1}\right)$. B: Double-grating setup at the third fraction Talbot distance $\left(D G_{3}\right)$. C: Single-grating setup at $40 \mathrm{~cm}$ propagation distance $(S G)$. The comparison of the three measurements shows strong corruption for $D G_{3}$ and $S G$ as consequence of phase-wrapping. At the border of the sample strong negative values occur (marked in blue). The resulting cupping-artefact is easily recognised by the rounded edged (marked in red).

corresponding material was taken as source for the MTF analysis. For a perfect spatial resolution this line would represent a step function. In the acquired experimental data, the edge of the material is always represented as slope, where a steeper slope corresponds to a higher spatial resolution. The MTF can be derived from the linear function and a certain cut-off frequency was taken to determine the spatial resolution using the voxel edge length. The Rayleigh criteria states that the resolution limit is reached at an $80 \%$ loss in peak to peak contrast. ${ }^{14}$ This is according to a modulation transfer value of $\approx 10 \%{ }^{15,16}$. Therefore the cut-off frequency to define the spatial resolution was selected at the MTF value of $10 \%$. From the simulations, a strong dependency of the spatial resolution on the investigated material is expected. Therefore, the resolution was determined separately for each material present in the phantom.

The results of the analysis are listed in Table 4. All calculated results show, that the resolution is strongly influenced by the examined material. The highest resolution can be achieved for the lightest material (PMMA \& PA 6.6). On the contrary the materials with the highest density ( $\mathrm{Al} \& \mathrm{Ti}$ ) give the lowest resolution. A better resolution of the single-grating setup is also proven in this table. Using the single-grating interferometer the resolution for every material is well below $10 \mu \mathrm{m}$, while three of four measurements with a double-grating setup cannot reach a resolution below $10 \mu \mathrm{m}$. Only the $D G_{3}$ measurement at $40 \mathrm{keV}$ could achieve a resolution below $10 \mu \mathrm{m}$ and also shows the characteristic material dependency. A reason for the coarse resolution of the $D G$-setups is supposed to lie in the presence of low-frequent image artefacts, which are present across the whole reconstruction. It is noticeable in the $S G$ results, that a higher energy provides a better spatial resolution. This can be easily explained by the ratio of increased distance (factor of 2) to decreased $\delta$-value (factor of 4 ) accompanied by a smaller refraction angle. This causes a reduced blurring caused by signal and therefore the better resolution. 


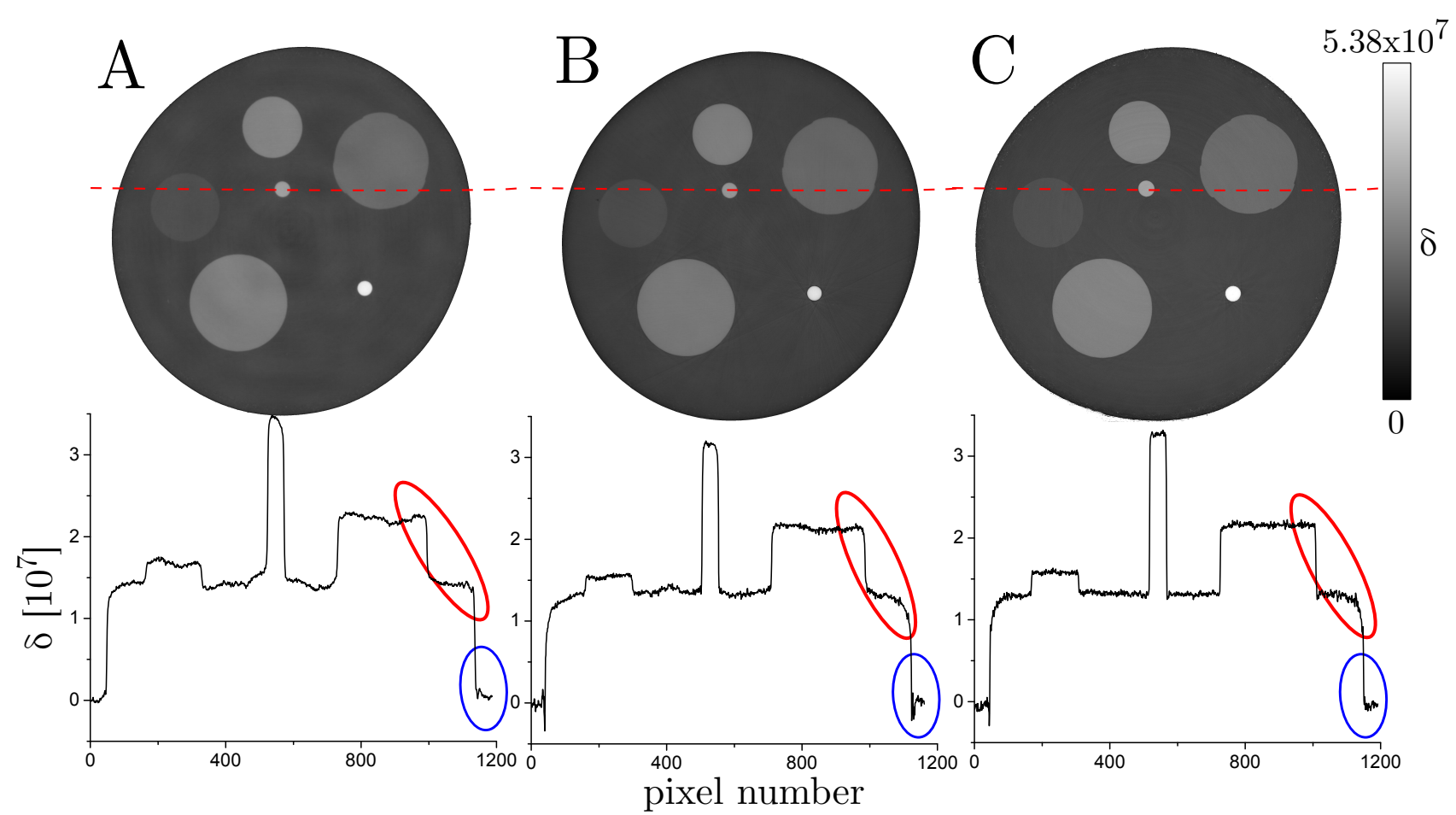

Figure 5: Reconstructed slices and line profiles of the test phantom at the different setups. A: Double-grating setup at the first fraction Talbot distance $\left(D G_{1}\right)$. B: Double-grating setup at the third fraction Talbot distance $\left(D G_{3}\right)$. C: Single-grating setup at $80 \mathrm{~cm}$ propagation distance $(S G)$. As for the measurement at $20 \mathrm{keV}, D G_{3}$ is still affected by phase-wrapping (marked in blue) and according cupping artefacts (marked in red). $S G$ also shows slight indication for phase-wrapping but seem to be not affected by cupping, as the profile shows sharp edges between the materials.

\section{SUMMARY AND DISCUSSION}

The experiments performed with the three different setups have been proven that all setups produce accurate images of the examined samples, on the condition that the reconstruction is not corrupted by strong phasewrapping artefacts. Phase-wrapping can result in a strong mismatch between the retrieved values and the literature values. The comparison of the signal to noise ratios have shown an advantage of the double-grating interferometer. The larger propagation distance of the $D G_{3}$-setup theoretically results in a clearly better SNRvalue than for the $D G_{1}$-setup. However, the occurrence of wrapping artefacts deteriorates the image quality, which in return decreases the SNR-values to about the same level as for the $D G_{1}$-setup. The SNR values for the $S G$-setup, which were expected to be between the values for the $D G_{1}$ and $D G_{3}$ setups, are much lower than expected. This can be explained by the very low visibility reached for this setup.

The resolution achieved with the single-grating setup exceeds the resolution with the double-grating interferometer for all measurements. With a spatial resolution down to $4 \mu \mathrm{m}$ this type of interferometer gives an excellent performance, where the actual spatial resolution is dependent on the investigated material. For lighter materials the resolution is in general higher and also can be improved with a higher energy. This proves again, that the achievable resolutions is strongly dependent on the materials properties rather than the experimental setup parameters.

With the double-grating interferometer a spatial resolution on the same length scale could be obtained, but the investigations have shown that this is highly dependent on the presence of reconstruction artefacts. Not merely wrapping artefacts, but rather instabilities of the interferometer like small grating vibrations have a major impact on the achievable spatial resolution. These instabilities also can cause a strongly disturbed background which is a big disadvantage for automatic image processing routines (e.g. segmentation). Due to the presence of only one grating and the larger period, the single grating interferometer is less influenced by this effect. 
Table 4: Spatial resolution for different phantom materials, determined by analysing the modulation transfer function. Values are given in $\mu \mathrm{m}$.

\begin{tabular}{|c|c|c|c|c|}
\hline & Material & $S G$ & $D G_{1}$ & $D G_{3}$ \\
\hline \multirow{6}{*}{ 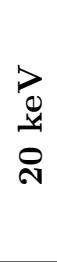 } & $\mathrm{PA}$ & 4.65 & 10.39 & 13.19 \\
\hline & PMMA & 4.84 & 10.76 & 11.43 \\
\hline & $\mathrm{Mg}$ & 5.35 & 10.13 & 18.32 \\
\hline & PCTFE & 8.60 & 10.91 & 16.78 \\
\hline & PTFE & 7.84 & 10.81 & 16.00 \\
\hline & $\mathrm{Al}$ & 6.59 & 11.82 & 19.20 \\
\hline \multirow{6}{*}{ 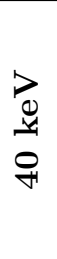 } & PMMA & 3.88 & —a $^{\mathrm{a}}$ & 5.82 \\
\hline & $\mathrm{Mg}$ & 4.76 & 14.71 & 7.19 \\
\hline & PCTFE & 5.22 & 16.20 & 8.05 \\
\hline & PTFE & 5.22 & 16.00 & 7.95 \\
\hline & $\mathrm{Al}$ & 5.77 & 15.80 & 9.41 \\
\hline & $\mathrm{Ti}$ & 6.04 & 14.71 & $5.02^{\mathrm{b}}$ \\
\hline
\end{tabular}

\section{ACKNOWLEDGMENTS}

First of all our gratitude goes to the Institute of Microstructure Technology of the Karlsruhe Institute of Technology, a partner in the Virtual Institute: 'New X-ray analytic methods in material science', for supply of all gratings necessary for this study. We would like to thank our first users from the Biomaterials Science Center of the University Basel for testing our new setup on biomedical applications.

The work was funded by the Helmholtz-Portfolio Theme Technology and Medicine.

\section{REFERENCES}

[1] Fitzgerald, R., "Phase-sensitive x-ray imaging," Physics Today 53(7), 23 (2000).

[2] Beckmann, F., Bonse, U., Busch, F., and Gnnewig, O., "X-ray microtomography (CT) using phase contrast for the investigation of organic matter:," Journal of Computer Assisted Tomography 21(4), 539-553 (1997$07)$.

[3] Momose, A., Kawamoto, S., Koyama, I., Hamaishi, Y., Takai, K., and Suzuki, Y., "Demonstration of x-ray talbot interferometry," Japanese Journal of Applied Physics 42, L866-L868 (2003-07-15).

[4] Davis, T. J., Gao, D., Gureyev, T. E., Stevenson, A. W., and Wilkins, S. W., "Phase-contrast imaging of weakly absorbing materials using hard x-rays," Nature 373(6515), 595-598 (1995-02-16).

[5] Reznikova, E., Mohr, J., Boerner, M., Nazmov, V., and Jakobs, P.-J., "Soft x-ray lithography of high aspect ratio SU8 submicron structures," Microsystem Technologies 14(9), 1683-1688 (2008-10).

[6] Mohr, J., Grund, T., Kunka, D., Kenntner, J., Leuthold, J., Meiser, J., Schulz, J., and Walter, M., "High aspect ratio gratings for x-ray phase contrast imaging," AIP Conference Proceedings 1466, 41-50 (2012).

[7] Greving, I., Wilde, F., Ogurreck, M., Herzen, J., Hammel, J., Hipp, A., Friedrich, F., Lottermoser, L., Dose, T., Burmester, H., Mller, M., and Beckmann, F., "P05 imaging beamline at petra III - first results," Proc. SPIE 9212 (2014).

[8] Haibel, A., Beckmann, F., Dose, T., Herzen, J., Ogurreck, M., Mller, M., and Schreyer, A., "Latest developments in microtomography and nanotomography at PETRA III," Powder Diffraction 25(2), 161-164 (2010-06).

[9] Bacher, W., Menz, W., and Mohr, J., "The LIGA technique and its potential for microsystems-a survey," IEEE Transactions on Industrial Electronics 42(5), 431-441 (1995-10).

\footnotetext{
${ }^{a}$ MTF-calculation did not succeed due to image artefacts and background noise

${ }^{\mathrm{b}}$ Due to phase-wrapping at the border of the Titanium rod the edge function is corrupted, leading to a false value.
} 
[10] Hipp, A., Beckmann, F., Lytaev, P., Greving, I., Lottermoser, L., Dose, T., Kirchhof, R., Burmester, H., Schreyer, A., and Herzen, J., "Grating-based x-ray phase-contrast imaging at petra III," Proc. SPIE 9212 (2014).

[11] Hipp, A., Herzen, J., Hammel, J., Lytaev, P., Schreyer, A., and Beckmann, F., "Single-grating interferometer for high-resolution phase-contrast imaging at synchrotron radiation sources," Proc. SPIE 9967 (2016).

[12] Barrett, J. F. and Keat, N., "Artifacts in CT: Recognition and avoidance," RadioGraphics 24(6), 1679-1691 (2004-11).

[13] Boas, F. E. and Fleischmann, D., "CT artifacts: Causes and reduction techniques," Imaging Med. 4(2), 229-240 (2012).

[14] Born, M. and Wolf, E., [Principles of Optics: Electromagnetic Theory of Propagation, Interference and Diffaction of Light], Pergamon Press (1959).

[15] Williams, D. and Burns, P. D., "Diagnostics for digital capture using MTF," PICS, 227-232 (2001).

[16] Loebich, C., Wueller, D., Klingen, B., and Jaeger, A., "Digital camera resolution measurement using sinusoidal siemens stars," Proc. SPIE 6502, 65020N-65020N (2007). 